\title{
Triton Tumor with Heterogeneous Cartilage Ingredient in Parotid Gland- An 88-Year Survey of the Literature on this Specially Malignant Tumor Hong $\mathrm{He}^{1,2 *}$, Xinyu Xia ${ }^{1,2}$, Zihao Zhang ${ }^{1,2}$, Ying Wang ${ }^{1,2}$, Xudong $\mathrm{Ma}^{3 *}$
}

${ }^{1}$ The Affiliated Stomatology Hospital, Zhejiang University School of Medicine, Yan'an Road, Hangzhou, China.

${ }^{2}$ Key Laboratory of Oral Biomedical Research of Zhejiang Province. Hangzhou China.

${ }^{3}$ Huzhou University, Huzhou China. honghehh@zju.edu.cn

*Co-Corresponding Authors: Xudong Ma, Hong He. Email: rainbow050607@126.com

\section{Abstract}

Malignant triton tumor(MTT) is a rare and highly malignant tumor, listed as a special type of malignant nerve sheath tumors, with an extremely poor prognosis and occurring at any age and in any occupation, usually in the head and neck, torso and upper limbs, et al. This report is a rare MTT with heterogeneous cartilage ingredient in parotid gland about its clinical and pathological features, differentiation diagnosis, treatments and classifications. Limited in clinical experience about this diagnosis and treatment, by reviewing literature, through domestic and foreign data, it is found that the incidence of MTT is showing an increasing trend in recent years. Phased surgery plus adjuvant therapy is a recommended choice. This is internationally a first report of the rare MTT with heterogeneous cartilage ingredient in parotid gland.

Keywords: Parotid gland, Malignant triton tumor, Cartilage, Clinical pathology.

\section{INTRODUCTION}

Malignant triton tumor (MTT) is a rare and highly malignant tumor with an extremely poor prognosis, less than 5 per cent in the total number of malignant nerve sheath tumors. The cancer can occur at any age and in any occupation, and usually occurs in the head and neck, trunk and upper limbs as well as other parts.

MTT derived its name from the experiment of Locatelli, in which he cut through the end of the sciatic nerve and implanted it into the soft tissue of the back of salamander, which induced the limb growth containing muscle and bone. He viewed that nerve sheath cells differentiated into muscle tissue under the influence of motor nerve fibers, and he considered that the nerve components of tumor can lead to a differentiation direction to skeletal muscle resembling with the normal nerves of amphibian salamander, so has the name " Malignant Triton Tumor " come in use ever since. MTT was reported for the first time in 1932 by Masson as a result of the potential to differentiate of a layer of embryo cells, that is, all the cells are poorly differentiated from the neural crest cells [1,2]. In 1992, WHO classification of soft tissue tumors lined it as a special type of malignant nerve sheath tumors, and called it malignant triton tumors or malignant nerve sheath tumors with rhabdomyosarcoma. In addition, there also exist clinical classifications including John's and International classification.

From statistics of 9,056 cases of cancer patients [3] during 52 years from January 1955 to December 2006, we found one case of this rare disease MTT, and considering no obvious new record has been found in the same region data. We further review and analyze here about the clinical features, pathological characteristics, diagnostic identification, treatment and classification of this special tumor disease reported at home and abroad within the scope of the search. By detailed study the data domestically and abroad, we find that the incidence of MTT in recent 
Triton Tumor with Heterogeneous Cartilage Ingredient in Parotid Gland- An 88-Year Survey of the Literature on this Specially Malignant Tumor

years is increasing, and that phased surgery plus adjuvant therapy program is a worthy choice. And this is the first reported MTT case, accompanied with heterologous cartilage components in the parotid gland, as a rare case.

\section{CASE REPORT}

A 45-year-old male patient was admitted to our hospital on November 6th, 2003, diagnosed as malignant triton tumor.

\section{History of his Illness}

More than two years ago, inadvertently, the 45-yearold male patient found that there was a soybeans size of painless mass in front of his left ear, no tenderness, no trismus, nor other discomfort. Two months ago, his left ear got a pain and the mucosal congestion, followed by a facial paralysis and left eye dysraphism, with his left corner of the mouth inclined to right, the mass increased, left nasolabial gully shallowed and wrinkles in his left forehead disappeared. At that time, he went to the Chinese medical hospital of Zhejiang Yongkang, accepted the acupuncture, Chinese medicine and anti-inflammatory drug treatment with no significant effect. He resorted to Yongkang City People's Hospital and accepted "the superficial lobe of the left parotid and tumor resection + grafting the facial nerve with the resected left great auricular nerve". Postoperative pathologic sections were sent to the Second Affiliated Hospital of Zhejiang University Medical College, and the pathology consultation reported it as malignant Triton tumor, then he was transferred to the hospital and was registered No.397277 for further treatment on November 6th, 2003. Upon registration, asymmetry was found in his bilateral facial and wrinkles in his left forehead disappeared. He was disable for wrinkles, with his left eye dysraphism, left nasolabial gully shallowed, his left corner of the mouth inclined to right, not able to make gills, an "S" shape Surgical scar in front of his left ear healed well, no palpable mass was found in his left parotid gland, no lymph node enlargement were found in his neck and beneath his bilateral jaw. Additional whole left parotid gland resection and cervical lymph node dissection were processed after he was transferred to the hospital. The pathology report was negative. Postoperative wound healed well. He discharged from hospital 15 days later, but died on November 25th, 2004 from recurrence and metastasis.

Laboratory data revealed the following values: the tumorwasS-100+,SMA+,MBP-,GFAP+/-,P63-,Desmin + , Myoglobin +. ( + is positively stained, - is negatively stained). The first postsurgically pathological report, which was a consulted report H03-0470 (figure 1,2) of Pathology Department in our Hospital according to Yongkang People's Hospital HE03-6564* 3, was "a malignant Triton tumor (malignant schwannoma with rhabdomyosarcoma differentiation and cartilage metaplasia) in the deep lobe of the left parotid gland". The second postsurgically pathological report 200313949 was "hypertrophic fibrous scar tissue in the left parotid gland region after the original surgery, no residual tumor components, negative with tumor components in residual parotid gland tissues, and 0 of 6 carotid lymph nodes from the left cervical substantial detection surgery was positive with tumor components.

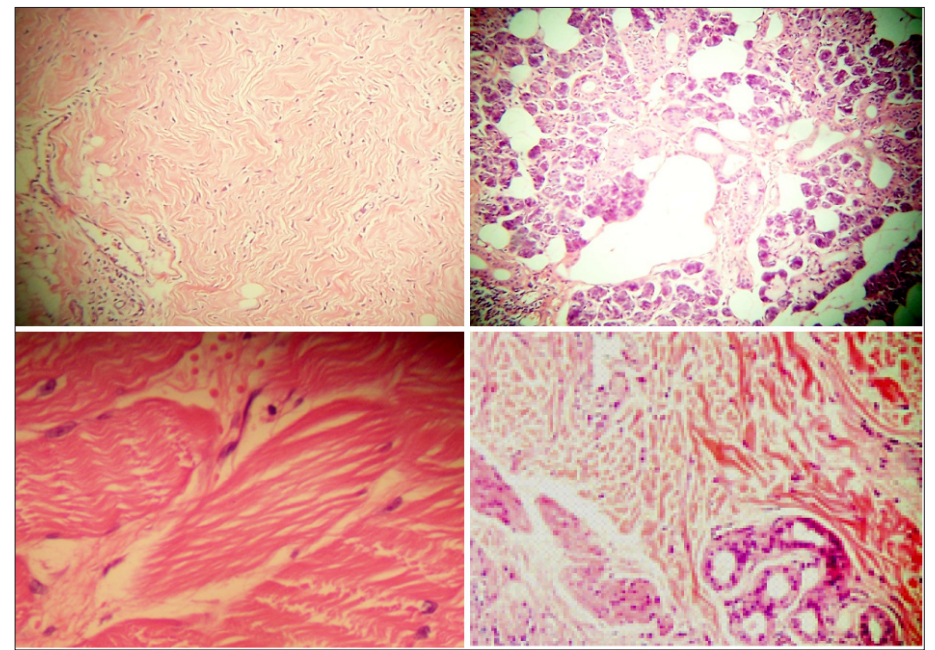

Fig 1. Optical microscopic pictures of the tumor. Saliva gland, and atypia rhabdomyo \& cartilages were observed. (HEx10, x20, x40, x20) 
Triton Tumor with Heterogeneous Cartilage Ingredient in Parotid Gland- An 88-Year Survey of the Literature on this Specially Malignant Tumor

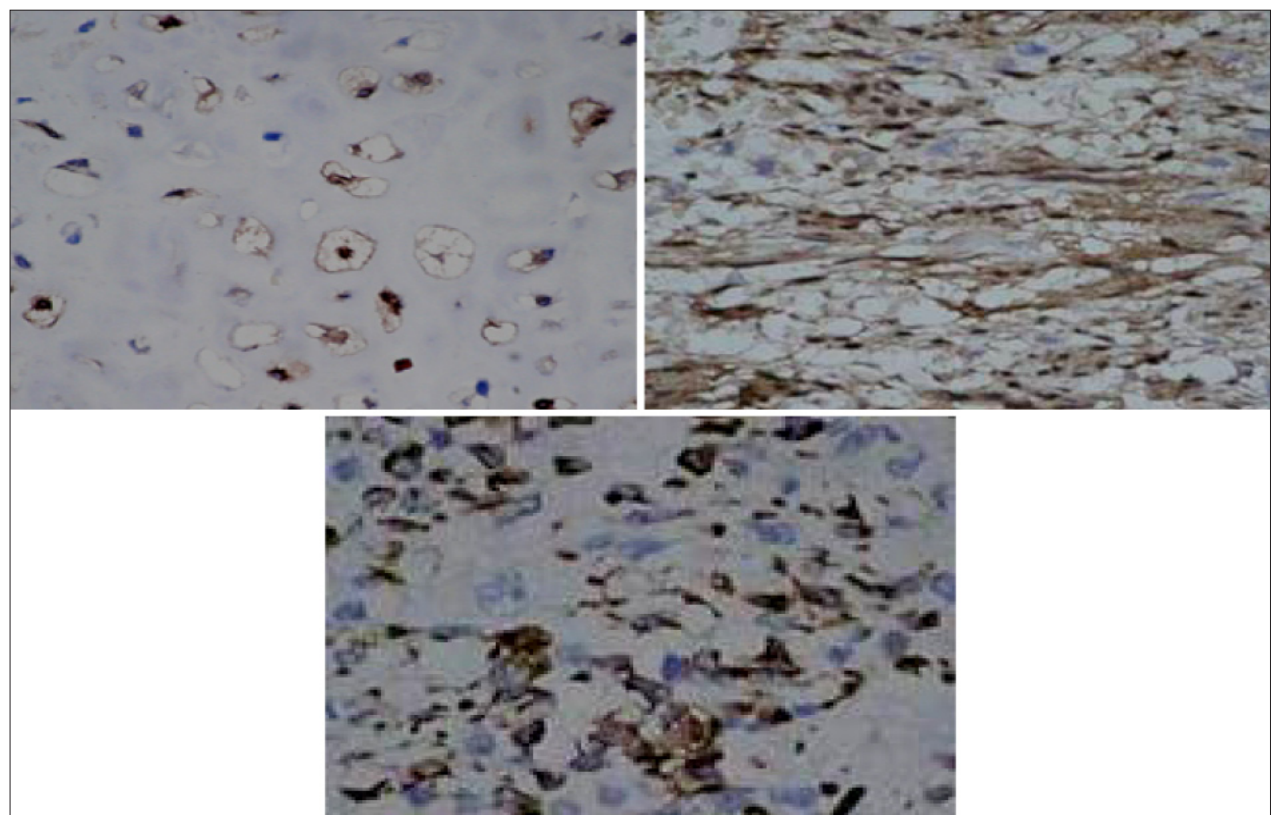

Fig 2. Optical microscopic immunohistochemical pictures of the tumor. Strongly positive stainings with Desmin and S-100 were observed, and cartilages were observed. (All x40)

\section{Comments}

This patient died from recurrence and metastasis after 4 years of his malignant triton tumor. This tumor has the following characteristics, (1) it can occur at any age and any occupation while the occurrence was rare. According to our detail study, the incidence of women was more in domestic than at abroad. It more usually occurred in people of 14 to 57 -year-old; the average was 33-year-old, being similar with the malignant nerve sheath tumors. there totally had 110 cases been reported before 2007 all over the world, which was consistent with Shen Yan Ying's statistics and reports [4], less than 5 percent of the total number of malignant nerve sheath tumors. Patients are getting to have larger age span, from 6-month to 80-year (mean 35-year), and there have been reported 53 cases abroad and 17 cases domestically according to 10 articles by 1997 [5], while 40 cases according to 37 articles during 1998-2007 domestically, in which 9 cases from 8 articles were reported during 19972001, and 31 cases from 29 articles during 20022007 [6] all only from China. 14 cases from 12 articles were during 2008-2010 domestically, 10 cases from 9 articles were in 2008-2010 abroad. During the first half year of 2011, 4 cases have been reported. To May 2012, about 170 cases were reported [7], and another 3 cases has been reported in July. 2013 [8]. And latestly 38 cases from 21 articles were from
Oct.2013.-.Sep.2019 domestically, 30 cases from 34 articles were from Oct.2013 - Sep.2019 abroad $[9,10,11]$ (Table 1, figure 3). This shows that there has been an increasing trend of the incidence rate in recent years. (2) It has an extremely wide range of tumor location including surface and internal organs, usually in the head and neck, trunk and upper limbs, also occurred in throat, buttocks, and retroperitoneal, having relationship with neural stem, with an easy recurrence locally and an early metastasis distantly. (3) It occur mostly in patients with neurofibromatosis and partly in patients with non-neurofibromatosis, with an extremly small number of occurrence in the differentiation into rhabdomyosarcoma of a original neurofibroma during its recurrence or metastasis $[12,13]$. According to whether there exists neurofibromatosis (Von Recklingbausch's disease), John divided the MTT into two groups: Group I, with neurofibromatosis, accounting for 70 per cent, usually occurred in young women, well to the head and neck, systemic multifocal brown spots after birth or at childhood, and then developed into a multiple mass, presented as typical neurofibromatosis performance. Spacing a few months or 20 years, single or multiple tumor growth suddenly accelerated, mass may also happen in the original non-tumor site, all present a malignant progression. The longest diameter of tumor ranges from 4.5 to $26 \mathrm{~cm}$, and most were large. This group can be accompanied with other tumors or 
Triton Tumor with Heterogeneous Cartilage Ingredient in Parotid Gland- An 88-Year Survey of the Literature on this Specially Malignant Tumor

deformities such as neuroblastoma, Wilms's tumor, and spinal defect. Group II, without neurofibromatosis, accounting for 30 percent, usually occurred in elder men, often in the trunk. No brown spots and multiple mass history, local in skin or subcutaneously, single or multiple, and often relate with the neural stem's direction. Correspondingly, the 45 years old male patient in this study with MTT occurred in his parotid gland, without neurofibromatosis, is classified as Group II by the John classification. And according to the International Classification of Diseases, the morphological coding for "malignant schwannoma with rhabdomyo cell differentiation" is M9561 / 3, which is named Terry tumor in Volume I of ICD-10 [14], a malignant tumor, and is a transliteration and free translation of "malignant salamander tumor". This can be checked in page 743 of Volume 3 of ICD-10 in accordance with its source of malignant neurogenic tumors, as well as its constitutes of nerve fiber cells and rhabdomyo cells.

Table 1. The number of reported cases of MTT in domestic China and abroad

\begin{tabular}{|l|l|l|l|}
\hline Duration & Domestic & Abroad & total \\
\hline $1932-1997$ & 17 & 53 & 70 \\
\hline $1998-2001$ & 9 & & 9 \\
\hline $2002-2007$ & 31 & & 31 \\
\hline $2008-2010$ & 14 & 10 & 24 \\
\hline $2011-2013.7$ & 20 & 19 & 39 \\
\hline $2013.8-2019.9$ & 38 & 30 & 68 \\
\hline
\end{tabular}

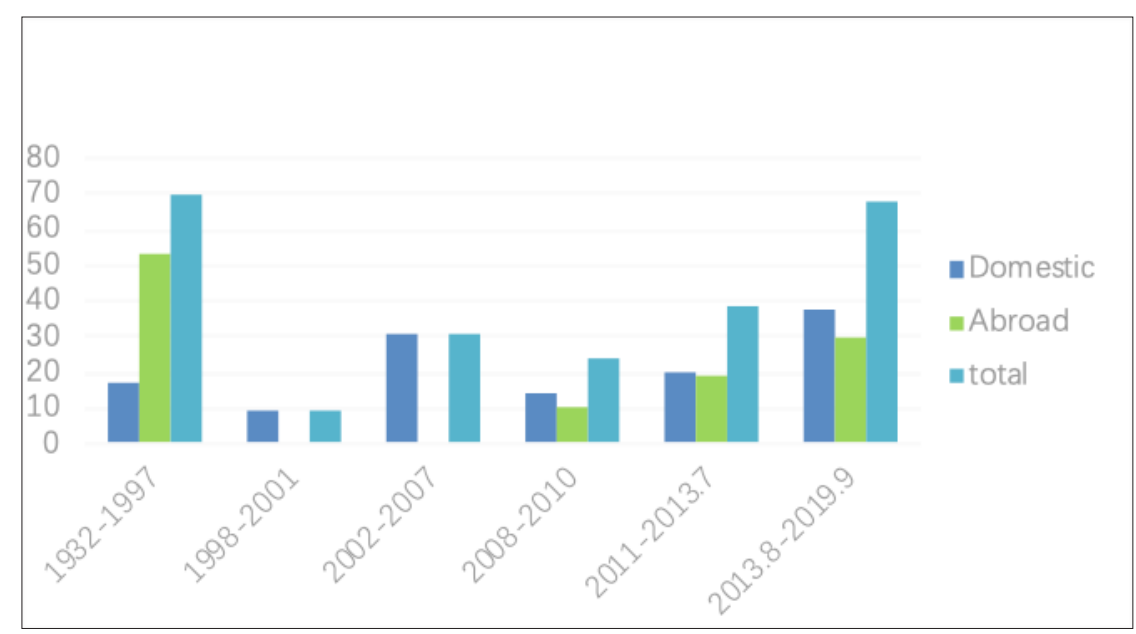

Fig 3. The number of reported cases of MTT in domestic China and abroad

In pathological view, the tumor generally presented as nodular, spindle or lobulated, hard and tough, mostly with a clear border, having or no having a false fibrous envelope. And the length of tumor was parallel or adhered to a neural stem. The section surface of tumor was grey-white or yellow, and mucus-like partly, with hemorrhage, necrosis and cystic lesion. A nodular section surface with a compound color of grey-while, grey-yellow and grey-brown and some saliva were observed in this case. And in microscopic examination, both rhabdomyosarcoma and malignant peripheral nerve tumor were observed. Small fibers were lined paralleling to each other among the cells of Schwannoma tumor cell (black stained with silver). Rhabdomyosarcoma could be identified by PTAH staining in addition to being yellow stained with VG, its cell morphology varied from being round or deformation as rhabdomyoblasts to being spindle with zoster-like cytoplasm. In addition, there also can be seen the single or multi-nuclear giant tumor cells with stripes in some cytoplasm.

Immunohistochemical Staining is of great help to the diagnosis of MTT [15], especially when stripes could not be examined under the optical microscopy, desmin and myoglobin positive can be used as a reliable basis for existence of the rhabdomyosarcoma. And when the tumor lack of the basis of connection with nerve fibers or it is not a malignant tumor from the nerve fibroma, S-100 is a marker of the Schwann cell, its positive staining support the existence of the malignant nerve 
Triton Tumor with Heterogeneous Cartilage Ingredient in Parotid Gland- An 88-Year Survey of the Literature on this Specially Malignant Tumor

sheath tumors [16,17] argued that a diagnosis of MTT should fit in with the following three conditions: (1) The tumor should derive from the peripheral nerve or type I of the neurofibromatosis. (2) The tumor should be composed mainly of malignant nerve sheath tumor components. (3) There are actually the rhabdomyoblast. During the joint monitoring of saliva CEA and CA-50, Hong, et al. [18] found its positive rate for detecting malignant tumors is high as $91.76 \%$, which can also provide a possible parameter for diagnosing MTT.

Most of the reports on treatment of MTT are radical surgery, supplemented with radiotherapy and chemotherapy, but with an uncertain efficacy and easy recurrence and metastasis. Andrej et al. [8] drew the conclusion that location of the primary tumor is a key factor for prognosis, which the view was consistented with Mijović et al.[19] , and surgical removal is the only treatment associated for survival rate. There is a lack of report on the conclusions of curative effects temporarily; there has been an indication that it is feasible to give systemic thermochemotherapy to children under keenly watch and proper treatment [20]. The malignance significantly increases when a malignant peripheral nerve sheath tumor is accompanied with heterogeneous components than ordinary type of it [2]. So MTT with heterogeneous components is of high malignence and poor prognosis.

This case had heterologous cartilage components, the degree of malignancy and the poverty of prognosis are serious, and it was only one year for him to survive after the enlarged removal of tumor and the cervical lymph nodes dissection refusing postoperative adjuvant radiotherapy or chemotherapy. It was reported that a phased surgery plus chemotherapy for a mediastinal MTT enlarged the follow-up survival time[21,22]. MTT has a recurrence rate of $43-100 \%[4,14,23,24]$, a transfer rate of $48 \%$, a 2 -year survival rate of $24 \%$, and a five-year survival rate of $12 \%$ to $26 \%$ postsurgically. There is no new report on the accurate survival rate in the past 10 years. This is a special report on malignant triton tumor with heterogeneous cartilage ingredient in man's parotid gland.

\section{ACKNOWLEDGMENT}

The work was supported by the Teaching Reform Project of Zhejiang University School of Medicine yxyb20172030. National and provincial Health Commission Co-construction Fund WKJ-ZJ-1623. National Health Commission Public Welfare Fund
(201502018). National Key R\&D Program of China 2016YFC0902702, Fund 2008C33026 [2011]666 from Science and technology Office of Zhejiang Province. 2016 Fund for 151 talents second level Zhejiang Province 2014-2017. Scientific Research Foundation for the Returned Oversea Chinese Scholar J20120036 from HR Office of Zhejiang Province. Baoshi Oversea Scholarship zups1507 2013-2015, China Scholarship Council [2009] 3004, and Research Program 2013KYB247 from Zhejiang Hygiene Bureau.

The authors would like to express their deep appreciation to vice director physician Jinkang Jia, Renal Department; pathologist Xin Gao and director pathologist Lirong Chen, Pathology Department, the Affiliated Second Hospital, Zhejiang University, School of Medical; director physician Jingrui Hu, Renal Department; and director physician Qingtao $\mathrm{Hu}$, Pathology Department, Yongkang People's Hospital, Zhejiang Province, for their sincerely enthusiastic help and support during preparation for the pathological data in this paper.

\section{REFERENCES}

[1] Jia H, Keiko Y, Marsha RR (2002). Characterization of a cysteine protinase inhibitor induced during neuronal cell differentiation. J Neurochem. 81: 922-934.

[2] Enzinger FM, Weiss SW (1988). Seft tissue tumors. Second edition. Mosby, University of Michigan. Michigan. 795pp.

[3] He H, Chen GF, Zhou Y, Zhang SZ (2007). Statistical Analysis of 9,056 Oral and Maxillofacial Tumors, Cysts and Tumor-like Lesions in 52 years. J Practical Oncol. 22: $532-535$ (in Chinese).

[4] Shen YY, Yin XL (2007). Clinical Pathological Analysis of mediastinal malignant triton tumor. J Shanghai Traffic Univ (Med). 27: 254-6 (in Chinese).

[5] Flanes F, Sanz-Oratega J, Suarez B, Sanz-Esponera J (1997). Triton tumor of the parotid area. Case report. Histol Histolpathol. 12: 51-56.

[6] Zhou Y, He H, Chen GF, Zhang SZ (2008). A malignant triton tumor of parotid gland with cartilage ingredient. J Practical Oncology. 23(6): 556-557 (in Chinese).

[7] Tian L, Shang HT, Bilal S, Li YP, Feng ZH, Lei DL, Cheng XB (2012). Treatment of malignant triton tumor in zygomatic region. J Craniofac Surg. 23(3): e265-268. 
Triton Tumor with Heterogeneous Cartilage Ingredient in Parotid Gland- An 88-Year Survey of the Literature on this Specially Malignant Tumor

[8] Mijović Z, Mihailović D, Zivković N, Kostov M, Zivković S, Stojanović N (2013). A rare case of retroperitoneal malignant Triton tumor invading renal vein and small intestine. Vojnosanit Pregl. 70(3): 322-325.

[9] Aykut B, Wieczorek K, Schirmacher P, Büchler M.W, Hoffmann K(2016). A rare case of recurrent malignant triton tumor in a male with NF1: Case report and mini-review. International Journal of Surgery Case Reports.21:121-124

[10] Merter A, Başarır K, Yıldız Y, Sağlık Y, (2017). Malignant triton tumor of the gluteal region in a patient unaffected by neurofibromatosis: A case report. Acta Orthopaedica et Traumatologica Turcica. 52: 236-239

[11] Soufiane G, Sandra F, Sébastien L, Jean-Charles Le Huec;(2017) Malignant triton tumor: Grand Round presentation of a rare aggressive case thoracolumbar spine tumor.European Spine Journal.28:1448-1452

[12] Dewit L, Albus-Lutter CE, De Jone AS Voûte PA (1986). Malignant sdwannoma with a rhabdomyoblastic component, a so-called triton tumor, A Clinicopathologic study. Cancer. 58: 1350-1356.

[13] Rose DS, Wilkins MJ, Birch R, Evans DJ (1992). Malignant peripheral nerve sheath tumor with rhabdomyoblastic and glandular diffenrentiation, Immunohistochemical Features. Histopathol. 21: 287-290.

[14] He JB (2004). Malignant triton tumor and ICD-10 Coding Analysis. China Cases. 5: 45 (in Chinese).

[15] Wang J, Weiss LM, Hu B (2004). Usefulness of immunohistochemistry in delineating renal spindle cell tumours. A retrospective study of 31 cases. Histopathol. 44: 462-471.

[16] Johuson TL, Lee MW, Meis JM (1994). Immuohistochemical characterization of malignant peripheral nerve sheath tumors. Am Surg Pathol. 4:121-135.

[17] Woodruff JM, Christensenw N (1993). Glandular peripheral nerve sheath tumors, Cancer. 72: 3618.

[18] He H, Chen GF, Zhou L, Liu YM (2009). A joint detection of CEA and CA-50 levels in saliva and serum of patients with tumors in oral region and salivary gland. J Cancer Res Clin Oncol. 135:13151321

[19] Andrej T, Beata B, Klaus W, Gratz, Sandro J, Stoeckli (2009). Prognostic factors for the malighant triton tumor of the head and neck. Wiley Periodicals, Inc Head Neck. 31: 679-688.

[20] Guan J, Liao YY, Ma R (2010). Systemic thermochemotherapy treated children triton tumor: one case report. World Health Digest. 07(26):201 (in Chinese).

[21] Lang LL, Pens F, Jancovici R (2003). Malignant "Triton" tumor of the posterior medlastinum: prolonged survival after staged resection. Ann Thorac Surg. 75: 1645-1648.

[22] Brooks, Euterline (1985). Maligrant "Triton" tumors natural history and immunohisto chemistry of nine new cases with literature review. Cancer. 55: 2543-2549.

[23] Daimaru Y, Hashimoto H, Enjoji M (1984). Malignant "triton" tumors: a clinicopathologic and immunohistochemical study of nine cases. Hum Pathol. 15: 768-778.

[24] Woodruff JM, Perino G (1994). Non-germ-cell or teratomatous malignant tumors showing additional rhabdomyoblastic differentiation, with emphasis on the malignant Triton tumor. Semin Diagn Pathol. 11: 69-81.

Citation: Hong He*, Xinyu Xia, Zihao Zhang, Ying Wang, Xudong Ma*. Triton Tumor with Heterogeneous Cartilage Ingredient in Parotid Gland- An 88-Year Survey of the Literature on this Specially Malignant Tumor. Archives of Dentistry and Oral Health. 2019; 2(2): 29-34.

Copyright: (C) 2019 Hong He, Xinyu Xia, Zihao Zhang, Ying Wang, Xu dong Ma. This is an open access article distributed under the Creative Commons Attribution License, which permits unrestricted use, distribution, and reproduction in any medium, provided the original work is properly cited. 